\title{
ОПЫТ ЛЕЧЕНИЯ БОЛЬНОГО С ЭКССУДАТИВНЫМ ПЕРИКАРДИТОМ, ВОЗНИКШЕГО НА ФОНЕ КОЛОРЕКТАЛЬНОГО РАКА
}

Доктор мед. наук, проф. Бойко В. В. ${ }^{1}$, доктор мед. наук, проф. Тамм Т. И. ${ }^{2}$, кандидат мед. наук Бучнева О. В. ${ }^{l}$

${ }^{I}$ ГУ «Институт общей и неотложной хирургии им. В.Т. Зайщева НАМН Украиныл», Харьков, Украина.

${ }^{2}$ Харьковская медицинская академия последипломного образования, Украина.

DOI: https://doi.org/10.31435/rsglobal_ws/31082019/6634

\section{ARTICLE INFO}

Received: 15 June 2019

Accepted: 10 August 2019

Published: 31 August 2019

\section{KEYWORDS}

colorectal cancer, metastases, pericarditis, pericardial effusion, cardiac tamponade.

\begin{abstract}
In the article presented the results of the treatment of pericardial effusion in a patient with sigmoid colon cancer. The pathological process in the pericardial cavity was asymptomatic and was discovered as an accidental find during a preoperative examination before surgery on the abdominal organs. The importance of timely diagnosis of such a serious and rare complication is shown. The presence of pericardial effusion in a patient with colorectal cancer changed the treatment tactics and forced to shift the focus of surgical interventions. This helped to reduce the risk of major surgical intervention on the abdominal organs and a favorable outcome for the patient.
\end{abstract}

Citation: Бойко В. В., Тамм Т. И., Бучнева О. В. (2019) Opyt Lecheniya Bol'nogo s Ekssudativnym Perikarditom, Voznikshego na Fone Kolorektal'nogo Raka. World Science. 8(48), Vol.2. doi: 10.31435/rsglobal_ws/31082019/6634

Copyright: (C) 2019 Бойко В. В., Тамм Т. И., Бучнева О. В. This is an open-access article distributed under the terms of the Creative Commons Attribution License (CC BY). The use, distribution or reproduction in other forums is permitted, provided the original author(s) or licensor are credited and that the original publication in this journal is cited, in accordance with accepted academic practice. No use, distribution or reproduction is permitted which does not comply with these terms.

Введение. Экссудативный перикардит с наличием избыточного накопления жидкости в полости перикарда может иметь различную этиологию. Метастатический экссудативный перикардит - редкое и очень опасное осложнение злокачественных новообразований. Согласно данным Европейской ассоциации кардиологов до 35\% всех перикардитов имеют неопластическую природу [1]. Целенаправленными исследованиями установлено, что около $10 \%$ больных со злокачественными новообразованиями имели избыточный объем жидкости в полости перикарда различной степени выраженности [1-3]. Экссудативный перикардит наиболее часто встречается у больных с онкологическим процессом в легких, молочных железах и злокачественными заболеваниями крови. Значительно реже скопление жидкости в перикарде встречается у больных с колоректальным раком и составляет $1,4-3 \%[1,8]$. В то же время заболеваемость колоректальным раком во всем мире непрерывно растет, как и количество его осложнений [5-7].

Накопление жидкости в полости перикарда происходит бессимптомно и обнаруживается как случайная находка при рентгенологическом или эхокардиоскопическом (ЭхоКС) исследовании. Появление симптомов сердечной недостаточности зависит от длительности и скорости накопления жидкости. Как правило, это происходит при значительном увеличении количества жидкости в полости перикарда и/или развитии тампонады сердца, что в таких случаях является абсолютным показанием для оперативного вмешательства $[1,3,4]$.

Учитывая низкую настороженность касательно возникновения такого осложнения у пациентов с колоректальным раком среди хирургов, которые акцентируют основное внимание непосредственно на лечении основного заболевания, возможно не определить наличие выпота в перикарде до операции. Вовремя не распознанный экссудативный перикардит может 
существенно усугубить, как течение основного процесса, так и привести к сдавлению сердца и летальному исходу $[1,5,6]$.

Цель исследования - представить случай своевременной диагностики экссудативного перикардита у пациента с раком сигмовидной кишки и показать последовательность и эффективность лечебной тактики.

\section{Материалы и методы.}

В ГУ «Институт общей и неотложной хирургии им. В.Т. Зайцева НАМН Украины» накоплен многолетний опыт лечения больных с заболеваниями желудочно-кишечного тракта, в том числе колоректальным раком. Во всех случаях диагноз устанавливали на основании анамнеза, общего осмотра, клинико-биологических, инструментальных методов исследования (УЗИ, КТ, ирригографии, колоноскопии), а также данных цитологических и гистологических исследований. В работе представлен клинической случай пациента с аденокарциномой сигмовидной кишки и наличием экссудативного перикардита.

Пациент У., 63 лет, поступил в клинику в плановом порядке с жалобами на снижение аппетита, затрудненный стул и вздутие живота, выраженную общую слабость. Считал себя больным в течение года. За медицинской помощью обратился впервые. При обследовании во время колоноскопии обнаружен инфильтративный процесс в области сигмовидной кишки с признаками ее сужения на одну треть просвета. Цитологическое исследование опухоли выявило наличие умеренно дифференцированной аденокарциномы. Из сопутствующих заболеваний у пациента имела место гипертоническая болезнь I-IIст.

В ходе предоперационного обследования по данным КТ обнаружен выпот в перикардиальной полости с диастазом листков перикарда более 2,0 см (рис. 1).

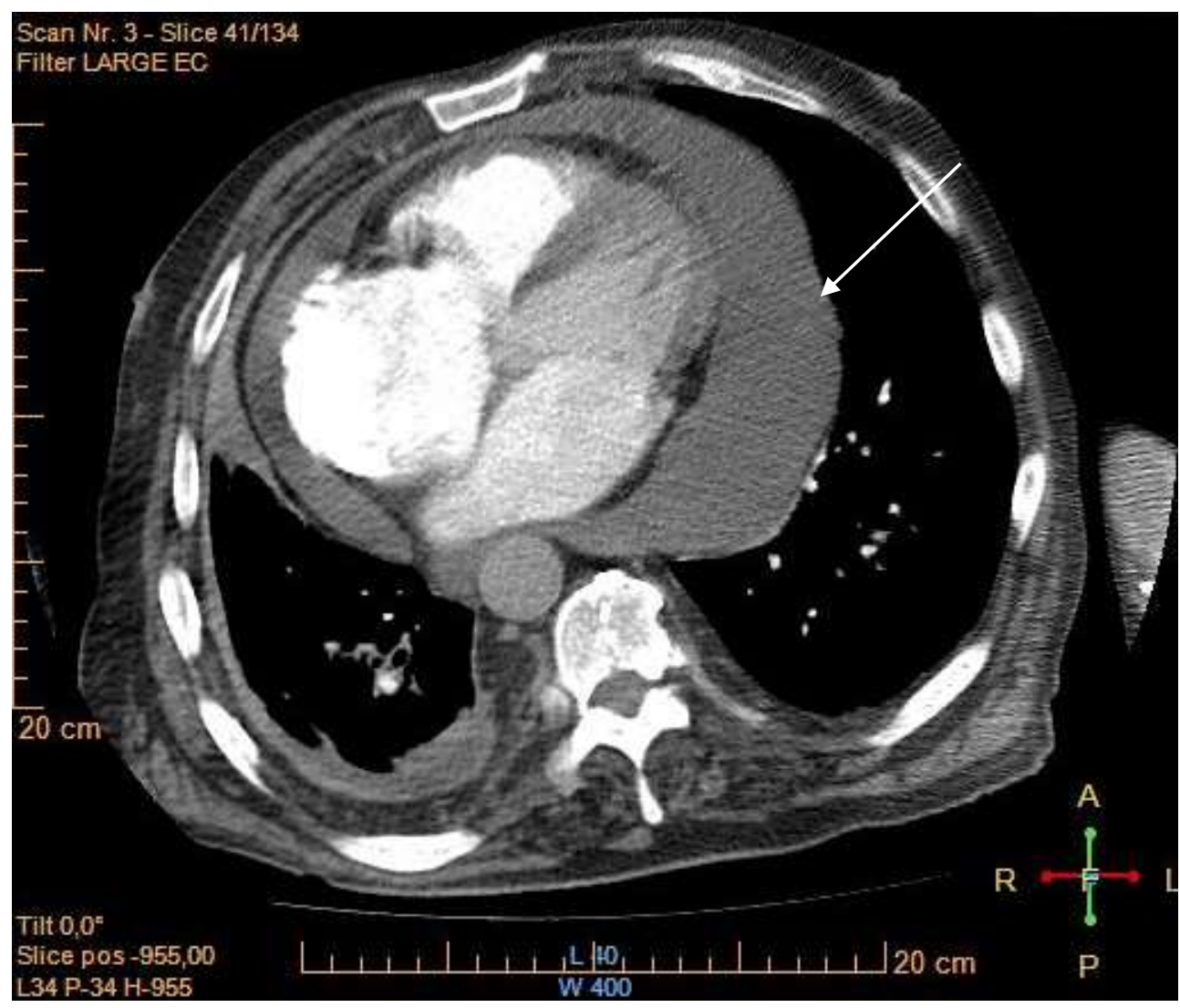

Рис. 1. КТ органов грудной клетки пациента У., 63 лет. Выпот в полости перикарда (указан стрелкой).

По данным выполненной ЭхоКС установлено наличие экссудативного перикардита с диастазом листков перикарда 2,0-3,0 cм, а также коллабирование правого предсердия с дискинезом межпредсердной перегородки. Нижняя полая вена коллабировала более чем на $50 \%$ (рис. 2). Со стороны клапанного аппарата сердца патологии не выявлено, значимой гипертрофии миокарда не обнаружено. Фракция выброса левого желудочка составила 55\%. 


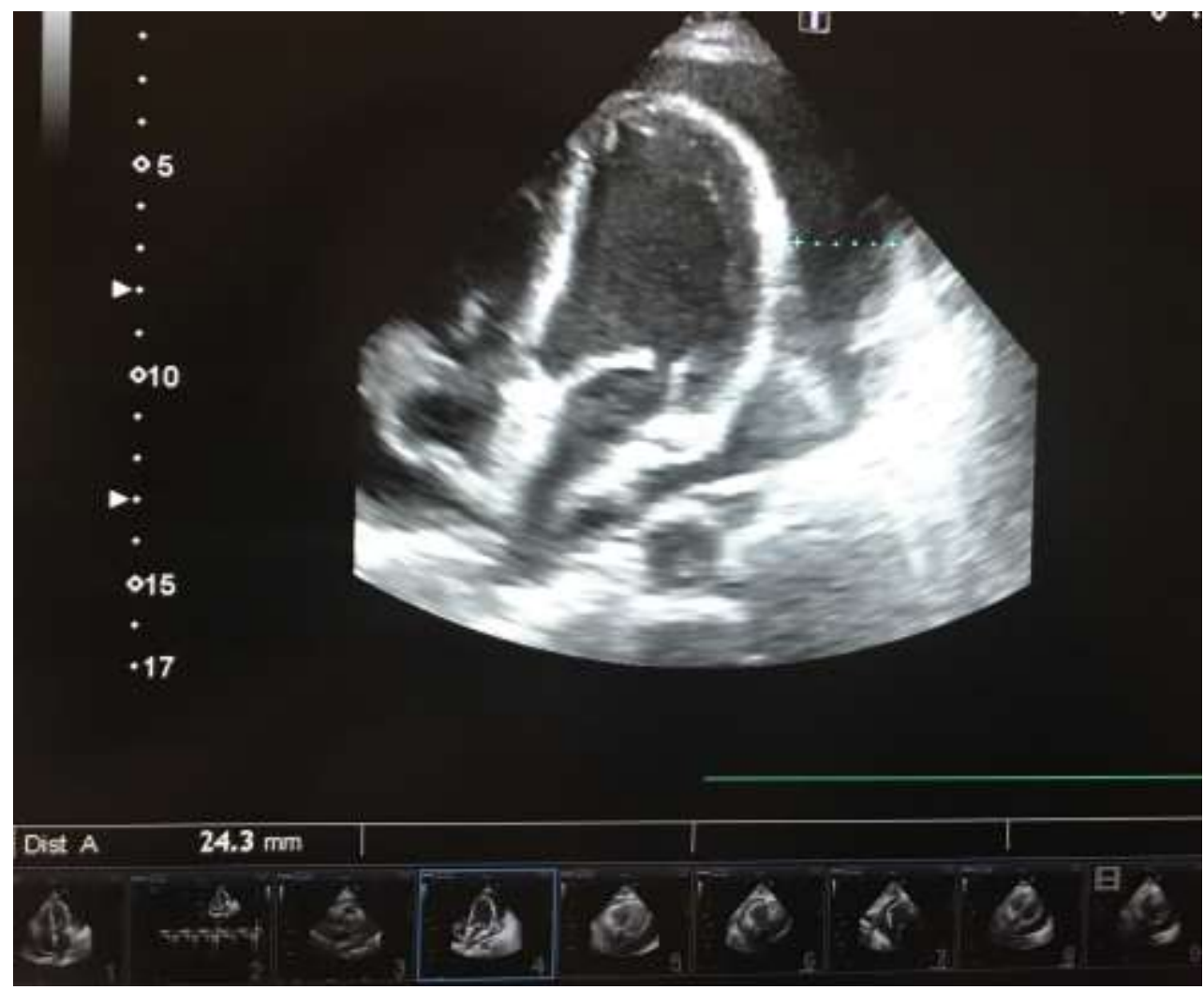

Рис. 2. ЭхоКС паџиента У., 63 лет. Экссудативный перикардит с диастазом листков перикарда 2,43 cм.

Учитывая отсутствие тампонады сердца, но наличие большого количества выпота в перикарде, а также явления начального сдавления сердца, что проявилось коллабированием правого предсердия с дискинезом межпредсердной перегородки, было принято решение о выполнении пункции и дренирования перикарда в качестве первого этапа лечения данного пациента.

Пункция перикарда была выполнена в условиях реанимации под динамическим мониторингом артериального давления, частоты сердечных сокращений и сатурации крови. В положении пациента полусидя, в условиях местной анестезии и под контролем ЭхоКС произведена пункция перикарда из подмечевидного пространства (по Лоррею). Одномоментно получено около 300 мл геморрагического отделяемого, которое было отправлено на цитологическое исследование. Для предотвращения развития острой сердечной недостаточности в полость перикарда был заведен дренаж по Сельдингеру диаметром $12 \mathrm{Fr}$ для последующего порционного и постепенного удаления выпота.

Всего по перикардиальному дренажу за 72 часа было получено 1700мл геморрагического отделяемого. При цитологическом исследовании в экссудате обнаружены атипичные клетки. Бактериологическое исследование жидкости из перикарда роста не выявило.

Дренаж был удален на 3 сутки после его установления, когда прекратился сброс отделяемого. После стабилизации общего состояния и восстановления полноценной сердечной деятельности выполнено основное оперативное вмешательство.

Учитывая тяжелое осложнение со стороны сердечно-сосудистой системы, больному выполнена открытая лапаротомия, во время которой обнаружена опухоль в средней трети сигмовидной кишки, прорастающая серозную оболочку $\left(\mathrm{T}_{3}\right)$. Одновременно выявлено увеличение отдельных лимфоузлов первого порядка. Произведена перевязка нижнебрыжеечной артерии и одноименной вены с забором измененных и обычных лимфоузлов и резекцией сегмента толстой кишки с опухолью. Операция завершена формированием толстокишечного анастомоза.

Послеоперационное течение со стороны брюшной полости протекало без особенностей. Больной на 9 сутки выписан домой. В последующем ему назначена химиотерапия по результатам гистологического исследования. В течение всего срока лечения пациенту выполняли контроль ЭхоКС для своевременного выявления рецидива экссудативного 
перикардита. После окончания стационарного лечения при контроле ЭхоКС через 1-3-6-12 месяцев данных, свидетельствующих о рецидиве перикардита не выявлено.

\section{Результаты и обсуждение.}

Анализируя приведенный случай, можно обратить внимание на то, что манифестация жалоб у больного была связана с наличием основного заболевания, а именно раком сигмовидной кишки. Отсутствие кардиальных жалоб и признаков нарушения дыхательной функции у данного пациента были связаны, вероятно, с постепенным накоплением выпота в перикарде, что позволяло стенкам последнего постепенно растягиваться, а сердечной деятельности быть в состоянии компенсации. Возникновение тампонады сердца возможно даже при незначительном количестве жидкости при резком и быстром ее накоплении в сердечной сумке. В данном случае даже при отсутствии тампонады сердца обнаружено большое количество выпота, а по данным ЭхоКС уже присутствовали начальные признаки сдавления сердца в виде коллабирования правого предсердия с дискинезом межпредсердной перегородки. Не смотря на наличие подтвержденной опухоли в сигмовидной кишке, оперативное вмешательство у пациента вначале выполнили на перикарде. Устранение экссудативного перикардита позволило избежать жизнеугрожающего состояния и в краткие сроки разрешить патологический процесс в сердце.

Стоит подчеркнуть, что наличие экссудативного перикардита независимо от количества жидкости в сердечной сумке создает дополнительный анестезиологический риск во время операции на органах брюшной полости в условиях общего обезболивания. Кроме того, в таких случаях является неоправданным проведение лапароскопических операций, когда возникает необходимость наложения пневмоперитонеума.

Экссудативный перикардит, как у данного больного, может длительное время существовать бессимптомно. Отсутствие своевременной диагностики могло привести в пери- либо послеоперационном периоде к развитию тампонады сердца. Учитывая редкость подобного осложнения у больных с колоректальным раком, настороженность на его счет среди хирургов очень низкая. Только тщательное плановое предоперационное обследование дает возможность диагностировать наличие экссудативного перикардита. Это позволяет сместить акцент хирургического воздействия и выполнить вначале оперативное вмешательство, направленное на ликвидацию жидкости в сердечной сумке. Такие действия позволяют снизить риск анестезиологического вмешательства и в целом благоприятно влияют на послеоперационное течение.

Выводы. У больных с колоректальным раком возможно развитие редкого осложнения в виде экссудативного перикардита, протекающего бессимптомно. Планирование и выполнение оперативных вмешательств в таких случаях надо начинать с санации сердечной сумки, что в целом благоприятно влияет на конечный результат.

\section{ЛИТЕРАТУРА}

1. ESC Guidelines for the diagnosis and management of pericardial disease / Y. Adler, P. Charron, M. Imazio [et al.] // Eur. Heart J. - 2015. - Vol. 36. - P. 2921-2964.

2. Ferrand F. Impact of primary tumor resection on survival of patients with colorectal cancer and synchronous metastases treated by chemotherapy: results from the multicenter, randomized trial Federation Francophone de Cancerologie Degestive 9601 / F. Ferrand, D. Malka, A. Bourredjem // Eur J Cancer. 2013. - Vol. 49, № 1. - P. 90-97.

3. Imazio M. Management of pericardial effusion / M. Imazio, Y. Adler // Eur. Heart J. - 2013. - Vol. 34. - P. 1186-1197.

4. Matijević D.N. Heamodynamic Monitoring of pericardial effusion with cardiac tamponade in a patient with colorectal cancer / Matijević D.N. // J Anesth Crit Care Open Access. - 2015. - Vol. 2(4): 00061. DOI: 10.15406/jaccoa.2015.02.00061.

5. Pericardial metastases in a long-surviving patient with sigmoid carcinoma / S. Lattuada, C. Saggia, G. Biaggi [et al.] // Tumori. - 2005. - №91. - P. 101-102.

6. Schmooll H. J. ESMO Consensus Guidelines for anagement of patients with colon and rectal cancer. A personalized approach to clinical decision maring / H. J. Schmooll, E. Van Cutsem, A. Stein // Ann. Oncol. - 2012. - Vol. 23, № 10. - P. 2479-2516.

7. Siegel R. Cancer statistics / R. Siegel, D. Naishadham, A. Jemal // CA Cancer J Clin. - 2012. - Vol. 62. - P. 10-29.

8. Surgical properties and survival of a pericardial window via left minithoracotomy for benign and malignant pericardial tamponade in cancer patients / S. Celik, M. Celik, B. Aydemir [et al.] // World Journal of Surgical Oncology. - 2012. - Vol. 10 (№123). - P. 1-8. 